\title{
Quality of life as an outcome measure
}

\author{
Christopher J Bulpitt
}

\section{Summary}

It is important to identify unknown and unquantified benefits and risks of therapeutic intervention that are reflected in the quality of life. This is especially important when the benefit : risk analysis is not clearly in favour of treatment. Quality of life is defined and target areas identified. The steps in the measurements of quality of life are discussed, including the validity, repeatability and sensitivity to change of existing methods; their acceptability, analytical problems; the presentation of the results; and the use of the results in economic evaluations. The Sickness Impact Profile, the Nottingham Health Profile and the Quality of WellBeing scale are compared for the assessment of patients with angina. The advantages and disadvantages of each is discussed together with the results from a doubleblind trial of treatment in angina where the Sickness Impact Profile was employed. Generic and disease-specific instruments are compared. The advantages and disadvantages of economic evaluations employing quality-of-life outcomes are discussed. Methods should be employed that withstand rigorous scientific evaluation. Both a health profile and a summary statistic such as a Health Status Index should be measured. If different treatments are to be compared, a randomised controlled trial should be employed. If the more expensive treatment is likely to be superior then costs should be collected and a cost-utility analysis performed.

Keywords: quality of life, outcome measure, benefit:risk ratio, Sickness Impact Profile

Division of Geriatric Medicine, Royal Postgraduate Medical School, Hammersmith Hospital, Du Cane Road, London W12 0NN, UK CJ Bulpitt

Accepted 30 October 1996
To qualify as an outcome measure, quality of life must be capable of definition and measurement and provide essential information to support evidence-based medicine. This evidence arises from the results of large randomised trials and tables 1 and 2 show the benefit : risk analysis for the treatment of mild hypertension determined from Medical Research Council (MRC) trials. ${ }^{1,2}$ An elderly person may be willing to risk an adverse effect of treatment over five years for a one in 75 likelihood of avoiding a stroke and a one in 100 chance of avoiding a cardiac event, but will a middle-aged person be prepared to take drug treatment for five years to prevent a 1 in 170 chance of a stroke and a 1 in 650 chance of a cardiac event? Possibly not, if he or she realises that with diuretic treatment there is the same probability as in the elderly of precipitating diabetes mellitus or gout; and with beta-blocker treatment still a high chance of lethargy, dyspnoea or Raynaud's phenomenon (table 2). Nevertheless, most physicians will urge treatment in the middle-aged for several reasons (see box 1).

A symptomatic assessment was made in the MRC trials but not a formal measurement of quality of life. If this had been done it may have considerably helped discussion on whom should be treated and with what. Such a measure would have to consider many aspects of quality of life to identify unknown or unquantified benefits and risks.

\section{The definition of quality of life}

One definition of health-related quality of life is the degree of subjective wellbeing attributable to or associated with lack of symptoms, psychological state and activities pursued. Box 2 gives a rough current consensus on what should be included under the heading of quality of life and those areas that are usually not considered. It may not be important that there is disagreement on content. The clinician is quite happy with a 'biochemical profile' of his patient. If this profile includes an item of no interest he or she ignores it; if an important and relevant piece of information is missing, for example, blood sugar, it is measured as an additional investigation. The same pragmatic attitude should be taken when measuring quality of life. However, as discussed below, the areas considered should be relevant to the problem at hand and however much a summary score is desirable, the underlying information should be sought and collated. The clinical chemist may rule that the biochemical screen is 'normal' but we need an estimate of how a result deviates from the expected value. The summary of 'normal' may hide the fact that some items have not been considered and others were 'borderline abnormal' rather than definitely normal.

\section{The measurement of quality of life}

The target areas listed in box 2 include physical and psychological well-being and activities. Nonmedical aspects are usually excluded, although costs to patients and carers may be assessed (see below).

Table 1 Benefits from active treatment compared to placebo in the two MRC trials of the treatment of mild hypertension ${ }^{1,2}$

\begin{tabular}{lcc}
\hline & Middle age & Elderly \\
\hline Rate reduction (per $10^{3}$ per year) & -0.1 & \\
All deaths & -1.2 & -0.8 \\
All stroke events & -0.3 & -2.7 \\
All cardiac events & & -1.9 \\
& & \\
Number to be treated to save one event over 5 years & 250 \\
All deaths & 2000 & 75 \\
All stroke events & 170 & 100 \\
All cardiac events & 650 & \\
\hline
\end{tabular}




\begin{tabular}{|l|}
\hline Assessment of benefits and \\
risks of treatment \\
\hline Table 1 underestimates the benefits of \\
treatment \\
- benefits are cumulative and may \\
increase as the patient ages; there \\
may be a time before benefits are \\
realised and the trials do not \\
continue long enough to fully \\
identify such benefits \\
trial results underestimate benefits \\
as 'high risk' placebo-treated \\
patients tend to be withdrawn and \\
given active treatment, for example \\
due to a rise in blood pressure \\
- the patient may be in a sub-group \\
expected to do particularly well with \\
treatment (eg, a male non-smoker in \\
the MRC trials) \\
Table 2 overestimates the risks of \\
treatment \\
- risks may not accumulate year on \\
year and are usually reversible on \\
stopping treatment \\
patients should be expected to pay \\
some costs in order to receive the \\
benefits (costs in financial terms or \\
adverse drug effects) \\
\hline
\end{tabular}

Box 1

\section{Assessment of quality of life \\ Areas to be assessed \\ - physical well-being measured by absence of symptomatic complaints: to include pain, system functioning (eg, respiratory, digestive, neurological), sleep, sexual functioning \\ - psychological well being: to include depression, anxiety, cognitive functioning \\ - activities: to include work, leisure, social functioning, fulfilment of roles (eg, mother, carer, etc) \\ Areas usually avoided owing to low probability of change in the short term \\ - financial security \\ - marital status \\ - freedom \\ - personality \\ Areas thought to be dicult to evaluate \\ - happiness \\ - ambition, expectation \\ - aggression, criminal activities \\ - burden \\ - religious experience, shame}

Table 2 Risks from active treatment compared to placebo in the two MRC trials of the treatment of mild hypertension. ${ }^{1,2}$ The lower figures for the numbers to be treated to get an adverse event assumes the risks are additive year on year and the high figures assume that risk is a one-off occurrence and not cumulative.

\begin{tabular}{|c|c|c|c|c|}
\hline \multirow[b]{2}{*}{ Diuretic } & \multicolumn{2}{|l|}{ Middle age } & \multicolumn{2}{|l|}{ Elderly } \\
\hline & $\beta$-blocker & Diuretic & $\beta$-blocker & \\
\hline \multicolumn{5}{|c|}{ Rate increase (per $10^{3}$ per year) } \\
\hline Diabetes mellitus & 4 & 0 & 4 & 3 \\
\hline Gout & 4 & 0 & 4 & 0 \\
\hline Lethargy & 2 & 6 & 4 & 17 \\
\hline Dyspnoea & 0 & 7 & 1 & 22 \\
\hline Raynaud's phenomena & 0 & 5 & 1 & 11 \\
\hline \multicolumn{5}{|c|}{ Number to be treated to get one adverse event over 5 years } \\
\hline Diabetes mellitus & $50-250$ & - & $50-250$ & $66-330$ \\
\hline Gout & $50-250$ & - & $50-250$ & - \\
\hline Lethargy & $100-500$ & $33-170$ & $50-250$ & $12-60$ \\
\hline Dyspnoea & - & $29-140$ & $200-1000$ & $9-45$ \\
\hline Raynaud's phenomena & - & $40-200$ & $200-1000$ & $18-90$ \\
\hline
\end{tabular}

Nine steps in the measurement of quality of life are given in box 3 . The methods must be valid (measure what they are supposed to measure), repeatable, sensitive to change (over time or as a result of treatment), and acceptable to the subjects. Ideally an instrument should not often produce a zero result (a floor effect) or a result of $100 \%$ (a ceiling effect) as this will limit the sensitivity of the measurement to change. A zero (good) result cannot improve and a $100 \%$ result cannot get worse. The weighting of the instrument should be acceptable, for example, the presence of a headache should not be equivalent to being confined to a wheelchair. Ideally, the instrument should have been employed previously so that problems have been identified, assessed and dealt with.

The presentation and analysis of quality of life results should be considered at the start of a study. A limited number of primary objectives will have to be defined in numerical terms (a given 'difference' to be detected at a significance level $\alpha$ and power 1- $\beta$ ). If a large number of independent outcome variables are to be presented then the significance levels will have to be adjusted for multiple results. Summarising the results and economic evaluation are discussed later.

\section{General measures of quality of life}

Three general measures of quality of life have been widely employed, the Sickness Impact Profile (SIP), ${ }^{3}$ the Nottingham Health Profile (NHP), ${ }^{4}$ and the Quality of Well-Being scale (QWB). ${ }^{5}$ Table 3 summarises some of the characteristics of these instruments.

These three instruments were compared in a study of 59 patients with angina. ${ }^{6}$ The age range was $46-79$ years, $73 \%$ were men and only $26 \%$ were employed or responsible for looking after the home. The majority of the patients were New York Heart Association (NYHA) grade II (42\%) and III (36\%), 17\% were grade I and only three (5\%) grade IV. The NYHA functional classification grades patients in one of four classes according to the degree of limitation of physical activity, as perceived by the physician. ${ }^{7}$ It has been extensively employed as an outcome measure in trials of treatment in congestive heart failure. ${ }^{8}$ The NYHA grade has a high inter-observer variability and is unlikely to be a very sensitive measure of outcome in clinical trials. Nevertheless, in the

Table 3 General measures of quality of life. Some of the characteristics of the Sickness Impact Profile (SIP), Nottingham Health Profile (NHP) and the Quality of Well-Being scale (QWB)

\begin{tabular}{|c|c|c|c|}
\hline & $S I P$ & $N H P$ & $Q W B$ \\
\hline Covers important areas? & yes & yes & yes \\
\hline 'In depth' enquiries? & yes & no & yes \\
\hline Number of items & 136 & 45 & varies \\
\hline Can be self-administered? & yes & yes & no \\
\hline Average time for completion (min) & 35 & 10 & 45 \\
\hline Valid and repeatable? & yes & yes & yes \\
\hline Floor and ceiling effects & minor & major & minor \\
\hline
\end{tabular}




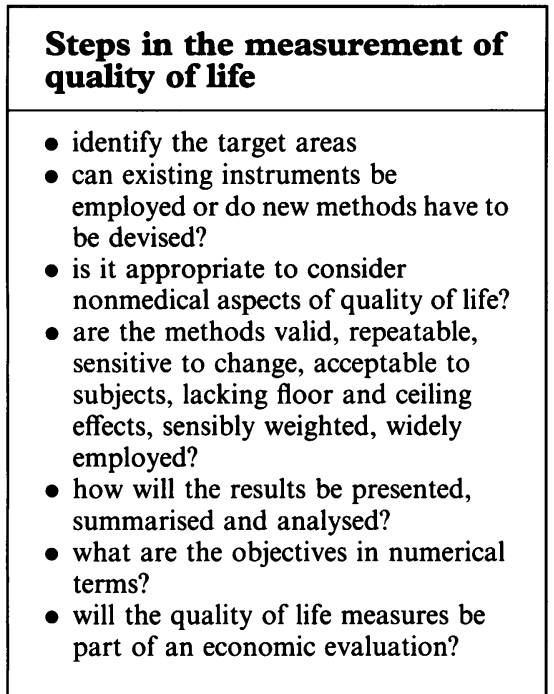

Box 3

\section{Problems in cost-utility analyses employing epidemiological data}

- epidemiological data may be unreliable and ungeneralisable.

- the fraction of the possible benefit to be produced by treatment may be unknown

- the fraction of benefit may change over time or with the duration of treatment

- the outcome measure, eg, quality-oflife-adjusted year of survival, may not have been measured but only estimated

- the scores given to a given utility may not be reasonable

- if the costs are accrued now and the benefits are observed later, the costs are greater and the benefits less than if the costs are incurred later and the benefits observed now. Both costs and benefits may have to be discounted (adjusted) for this temporal effect and it is difficult to decide on whether to make this adjustment and to what extent
Table 4 Multiplicative increase in scores when comparing NYHA (New York Heart Association) Grade III with I

\begin{tabular}{lll}
\hline Problem with & $S I P$ & $N H P$ \\
\hline Energy & - & $3.7^{\star}$ \\
Mobility & 3.1 & $4.6^{\star}$ \\
Ambulation & $4.3^{\star \star}$ & - \\
Body care & $3.4^{\star}$ & - \\
Home maintenance & 2.1 & $\infty{ }^{\star}$ \\
Recreation and pastimes & 2.1 & 2.9 \\
Social interaction & $2.3^{\star}$ & $\infty 0^{\star}$ \\
Social life & - & 1.2 \\
Home life & - & $\infty$ \\
Sex life & - & 0.72 \\
Eating & 1.5 & - \\
Communication & $18.2^{\star \star}$ & - \\
Pain & - & $4.7^{\star}$ \\
Sleep and rest & 1.2 & 0.93 \\
Holidays & - & 1.3 \\
Emotion & 3.0 & 3.8 \\
Alertness behaviour & $4.0^{\star}$ & - \\
Physical score & $3.6^{\star \star}$ & - \\
Psychosocial score & $3.1^{\star}$ & - \\
TOTAL & $2.7^{\star}$ & - \\
\hline
\end{tabular}

${ }^{\star} \mathrm{p}<0.05,{ }^{\star \star} \mathrm{p}<0.01$. SIP $=$ Sickness Impact Profile, NHP=Nottingham Health Profile, $\infty=$ infinite increase

study of 59 angina patients the gradings I to III were employed as a 'gold standard' to see which quality-of-life measure distinguished most clearly between the grades. The QWB scale was found to be less useful than the SIP or NHP for the following reasons: it always needs an interviewer, it is lengthy and difficult to administer, the overall score is dominated by the symptom scores and a single symptom is rated as the most distressing. In this study breathlessness was often the most distressing symptom, making the QWB scale insensitive to changes in chest pain. ${ }^{6}$

Table 4 gives the increase in the profile scores for different dimensions of the SIP and NHP on comparing NYHA grade III with I. This increase in grade was associated with a 3.7 -fold $(p<0.05)$ increase in problems with energy on the NHP and a 4.3-fold increase $(p<0.01)$ in problems with ambulation on the SIP. The two profiles compared well in this assessment, although the SIP had the advantage of being able to produce total physical, psychosocial and overall scores. The SIP consists of 136 questions and takes $30-40$ minutes to complete against 38 questions for the NHP, taking only about 10 minutes. The benefits of the extra effort were examined for social interaction, emotion and physical mobility in the three NYHA grades by determining the coefficient of variation. This was lower for the SIP than the NHP in eight of the nine comparisons and the median result was zero on six classifications with the NHP, giving troublesome 'floor effects'. The SIP is therefore likely to be more sensitive to change than the NHP and a trial including SIP will require less patients to demonstrate a given difference between treatments. Although the NHP is quicker to complete and very useful for studies of pain, ${ }^{9}$ the SIP was chosen for the final trial, ${ }^{10}$ in which it was shown that tolerance to glyceryl trinitrate by continuous application rendered the benefits undetectable whereas tolerance to the adverse effect of headache was less marked, leading to a lower quality of life on active treatment.

Many researchers are replacing the three general or generic measures discussed above with the Short-Form 36-item (SF-36) instrument. ${ }^{11}$ The SF36 has undergone a rigorous development but proof is required that 36 questions can replace the 136 questions of the SIP without loss of sensitivity to change or the emergence of floor and ceiling effects.

\section{Generic or disease-specific instruments?}

The SF-36 has been employed to identify changes associated with the concentration of glycosylated haemoglobin in diabetic subjects. It failed to detect changes and the authors suggested that a disease-specific measure of quality of life may have displayed a stronger relationship with glycaemic 


$$
\text { Cost }- \text { utility }=\frac{\mathrm{C}-\mathrm{B}}{\mathrm{U}}
$$

where $C=$ costs (direct and indirect), $B=$ economic benefits (direct and indirect), $U$ = life gain, adjusted for quality, eg, QALY (quality-adjusted life year), HYE (healthy years equivalent). (QALY $=$ life years gained $\times$ Health Status Index)

Figure Cost-utility analysis

\section{Using quality of life as an outcome measure}

- the measure of quality of life should include the areas relevant to the condition under investigation

- the methods should be valid, repeatable, sensitive to change, acceptable, and lacking floor and ceiling effects

- when possible, instruments should be used that have been widely employed and investigated

- both a Health Status Index and a health profile should be obtained

- when the effects of different treatments are to be compared, this should be done in a randomised controlled trial, double-blind if possible

- in a randomised controlled trial, if the more expensive treatment is likely to be the best treatment then costs should be collected and a costutility analysis performed

\section{Box 5}

1 Medical Research Council Working Party. MRC trial of treatment of mild hypertension: principle results. $B M \mathcal{F}$ 1985; 2: 97-104.

2 Medical Research Council Working Party. MRC trial of treatment of hypertension in older adults: principle results. BMF 1992; 304: 405-12.

3 Bergner M, Bobbitt RA, Carter WB, Gilson BS. The Sickness Impact Profile: development and final version of a health status measure. Med Care 1981; 19: 787-805.

4 Hunt SM, McKenna SP, McEwan J, Backett

EM, Williams J, Papp E. A quantitative approach EM, Williams J, Papp E. A quantitative approach to perceived health status: a validation study.

5 Kaplan RM, Bush JW, Berry CC. Health status: types of validity for an index of well-being. Health Serv Res 1976; 11: 478-507.

6 Visser MC, Fletcher AE, Parr G, Simpson A, Bulpitt CJ. A comparison of three quality of life instruments in subjects with angina pectoris: the Sickness Impact Profile, the Nottingham Health Profile, and the Quality of Well Being Scale. $\mathcal{f}$ Clin Epidemiol 1994; 47: 157-63.

7 The Criteria Committee of the New York Heart Association. Diseases nomenclature and criteria for diagnosis. Boston: Little Brown, 1964; por diagn $110-3$.

8 Lipkin D, Poole-Wilson PA. Treatment of chronic heart failure: a review of recent drug trials. $B M F$ 1985; 291: $993-6$. control. ${ }^{12}$ Similarly, when measuring quality of life in mild heart failure, the generic methods may be less successful than a disease-specific measure such as the Minnesota Living with Heart Failure questionnaire. ${ }^{13-15}$ However, it is difficult to differentiate between the lack of sensitivity of an instrument and the fact that quality of life changes very little or not at all.

In many fields a disease-specific instrument has not been developed and tested. In such a circumstance the adoption of available and relevant generic measures has proved successful. Examples are provided in the field of hypertension. ${ }^{16,17}$

\section{Health Status Index (HSI) or profile?}

A HSI is a summary score that encompasses all the quality-of-life data. It usually ranges from 0 (dead) to 1 (perfect health) and is employed in the QWB scale. A health profile is a set of scores describing different dimensions of quality of life such as provided by the SIP and NHP. The advantages of the HSI is that it summarises the data and can be employed to adjust the survival on treatment for the quality of life on that treatment. For example, if the cost of obtaining an extra year of life is $£ 5000$ with a quality of life of only 0.5 , then the cost of a quality-of-life-adjusted year of survival (a QALY) would be $£ 10000$. The calculation is given in the figure. If information on costs and quality of life were obtained within a randomised controlled trial comparing two treatments then this would be a very valuable outcome measure comparing the two drugs. Unfortunately this has not been done and inappropriate comparisons have been made of treatments that improve quality of life but not survival (such as a hip replacement), and treatment that improves survival but not quality of life, such as renal dialysis. ${ }^{18}$ Such comparisons have led to severe criticisms of QALYs, ${ }^{19}$ although proper comparisons could be most useful. Box 4 lists some of the problems encountered when trying to perform cost-utility analyses on epidemiological data. ${ }^{20}$

If a HSI is reported as an outcome measure it must be supplemented by detailed information such as provided by a profile. If a report is to be accepted the reader must see not only the direction that quality of life has changed but why and how. The clinician and researcher require to see precisely the areas of quality of life that are affected, while the policy-maker is interested in the cost effectiveness of the treatment in standardised units.

\section{Conclusions}

Box 5 gives six recommendations for the use of quality of life as an outcome measure. The measure must be relevant, scientifically sound, have been extensively tried and tested, and include both a profile and a summary statistic. Different treatments should be compared in a randomised controlled doubleblind trial and the collection of costs considered if a cost-utility analysis is planned. A major current problem is that, although our instruments for measuring quality of life may differentiate a patient with hypertension from one with insulin-dependent diabetes mellitus, we require methods that are sensitive to the changes brought about by treatment. The challenge is to identify or develop these sensitive instruments.

9 Parr G, Darekar B, Fletcher AE, Bulpitt CJ. Joint pain and quality of life; results of a randomised trial. Br f Clin Pharmacol 1988; 27: 235-42.

10 Fletcher AE, McLoone P, Bulpitt C. Quality of life on angina therapy: a randomised controlled trial of transdermal glyceryl trinitrate against placebo. Lancet 1988; ii: 4-8.

11 Ware JE, Sherbourne CD. The MOS 36-item short-form health survey (SF-36): conceptual framework and item selection. Med Care 1992; 30: 473 .

12 Weinberger M, Kirkman MS, Samsa GP, et al. The relationship between glycemic control and health-related quality of life in patients with noninsulin-dependent diabetes mellitus. Med Care 1994; 32: 1173-81.

13 Rector TS, Kubo SH, Cohn JN. Patients' self assessment of their congestive heart failure: II content, reliability and validity of a new measure - the Minnesota Living with Heart Failure Questionnaire. Heart Fail 1987; 3: 198-209.

14 Kubo SH, Gollub S, Bourge R, et al. for the Pimobendan Multicenter Research Group. Beneficial effects of pimobendan on exercise tolerance and quality of life in patients with heart failure: results of a multicenter trial. Circulation 1992; 85: $942-9$.
15 Rector TS, Kubo SH, Cohn JN. Validity of the Minnesota Living with Heart Failure Questionnaire as a measure of therapeutic response: effects of enalapril and placebo. Am $\mathcal{f}$ Cardiol 1989; 64: $50-5$.

16 Croog SH, Levine S, Testa M, et al. The effects of antihypertensive therapy on the quality of life. $N$ Engl $\mathcal{F}$ Med 1986; 314: 1657-64.

17 Fletcher AE, Bulpitt CJ, Chase DM, et al. Quality of life with three antihypertensive treatments - cilazapril, atenolol, nifedipine. Hypertension 1992; 19: 499-507.

18 Maynard A. Requirements for health care purchasers. In: Walker SR, Rosser RM, eds, Quality of life assessment: key issues in the 1990s. The Netherlands: Kluwer Academic Publishers, 1993; pp 413-26. cost: a review of cost-effectiveness analysis. $\mathcal{f}$ Hypertens 1991; 9: 193-8.

20 Johannesson $M$, Jönsson B. Cost-effectiveness analysis of hypertension treatment: a review of methodological issues. Health Policy 1991; 19: 55-78.
19 Fletcher A. Pressure to treat and pressure to 